\title{
Réécriture et histoire dans Ulysse de Joyce
}

\author{
Mendel Péladeau-Houle \\ Université d'Ottawa
}

Les thématiques de la réécriture et de l'histoire dans le roman Ulysse de James Joyce ont été on ne peut plus traitées. D’une part, le roman affiche un titre qui explicite d'emblée une filiation à l'Odyssée d'Homère. De l'autre, l'histoire est sans doute l'une des principales thématiques de l'œuvre de Joyce ${ }^{1}$. Les deux thèmes n'ont, cela dit, jamais été étudiés de concert. Le premier est certes celui qui a été examiné le plus prolixement. Bien que plusieurs hypothèses aient été proposées pour expliquer la réécriture dont fait état le roman, deux d'entre elles peuvent être considérées comme majoritaires. La première met en avant l'incompatibilité des époques des deux textes (Levin,

${ }^{1}$ Qui l'aborde peut-être avec plus de clarté encore dans Finnegans Wake. 
1960, p. 71-73; Pound, 1968, p. 406; Madtes, 1983, p. 30; Levitt, 1999, p. 32 et 55). La deuxième présente l'Odyssée comme un ressort narratologique fertile (Ellmann, 1962, p. 423; Tagopoulos, 1992; Friedman, 1993; Norris, 1998). En nous éloignant de ces deux approches canoniques, nous souhaitons proposer, dans le cadre de cette étude, que la réécriture ${ }^{2}$ puisse être considérée à partir de la conception joycienne de l'histoire. L'article se divisera en trois parties. La première s'attardera à présenter le rapport conflictuel entre le réel et l'histoire dans le roman. La deuxième montrera comment la réécriture peut être considérée comme une extension de cette problématique. La troisième, finalement, fera l'analyse d'une thématique ponctuelle du roman, celle de la nostalgie, dont il s'agira de montrer que sa parodie, sur le plan de la réécriture donc, constitue une critique larvée de l'histoire. Bien que multipliant les approches, l'article gardera néanmoins un point de mire unique : montrer que l'analyse de la réécriture dans Ulysse se trouve éclaircie par celle, connexe, de l'histoire.

\section{Le réel et l'histoire}

Au tout début d'Ulysse, Stéphane Dedalus, dit, on s'en souvient : «L'Histoire [...] est un cauchemar dont j'essaie de m'éveiller. ${ }^{3}$ » (p. 38) L'aphorisme, qui résume la conception joycienne de l'histoire, la présente comme une déréalisation du réel, dont le caractère péjoratif est renforcé par le terme «cauchemar »

\footnotetext{
2 Plusieurs études montrent qu'Ulysse est le fruit de plusieurs hypotextes. Nous nous limiterons cependant à l'Odyssée dans le cadre de cet article.

${ }^{3}$ Le segment coupé est négligeable : "L'histoire, dit Stephan Dedalus, est un cauchemar dont j'essaie de m'éveiller. »
} 
plutôt que «rêve ». Le personnage, dont l'état a priori est un état de facticité du rapport au réel, serait ainsi mû par ce que l'on pourrait appeler une volonté de dé-déréalisation du réel, lui permettant un véritable rapport à la vie. L'épisode que le schéma Linati ${ }^{4}$ intitule «Éole » présente cependant un pendant à l'apophtegme initial qui remet en cause cette capacité du personnage à s'extraire de l'histoire : « - Il leur a servi ça tout chaud, dit Myles Crawford, toute la sacrée sanglante histoire. / Cauchemar dont tu ne pourras jamais t'éveiller. » (p. 154) Toute la complexité de la conceptualisation de l'histoire chez Joyce se trouve entre ces deux pôles : entre volonté et incapacité du personnage à mettre l'influence néfaste de l'histoire entre parenthèses, de sorte à pouvoir accéder à une existence plus authentique.

Dans James Joyce and the Language of History, Robert Spoo suggère que la thématique de l'histoire dans Ulysse soit liée à ce que "[Friedrich] Nietzsche appelle la "maladie de l'histoire" $"$ " (p. 6). Dans la deuxième partie des Considérations inactuelles, intitulée De l'utilité et de l'inconvénient des études historiques pour la vie, en effet, Nietzsche met en garde contre ce qu'il nomme les "excès du sens historique dont souffre le présent» (p.365). Ainsi, bien que chez Joyce, comme chez Nietzsche, l'histoire soit conceptualisée à divers niveaux, il convient avant tout de la définir, chez l'un comme chez l'autre, à

\footnotetext{
${ }^{4}$ Le schéma Linati est un guide de lecture envoyé par Joyce à son ami Carlo Linati et qui découpe notamment le roman en épisodes selon ceux qui composent son hypotexte, l'Odyssée d'Homère.

5 Je traduis : "I argue that Stephen's "nightmare" and the text of Ulysses itself are distinct but related responses to what Nietzsche called "the malady of history", the cultural obsession with the past and with the explanatory power of historiography, which, Nietzsche believed, was destroying intellectual and moral health in the nineteenth century. »
} 
travers son rapport à la vie et, plus particulièrement, à travers les effets délétères qu'elle a sur celle-ci. L'histoire ne se pense comme science du passé que subsidiairement: elle est avant tout une réalité pragmatique des société et sujet présents. Notons d'autre part que la critique a relevé la diversité des héritages et influences historiques dans le roman: grécoromain, irlandais, chrétien, etc. Elle a aussi souligné ses conséquences sur la vie des personnages: machisme, antisémitisme, stigmatisation de la sexualité, etc. (Nadel, 1989; Froula, 1991; Fairhall, 1993).

Ce combat de restitution de la vie, qui passe par le rejet de l'histoire, se joue avant tout chez Joyce à travers la figure du personnage. Les personnages ont en effet conscience d'une sorte de devoir de rupture avec l'histoire. Dans les premières pages d'Ulysse, par exemple, Buck Mulligan raille le refus de Stéphane Dedalus d'obtempérer aux dernières volontés de sa mère, qui souhaitait le voir s'agenouiller et prier pour elle à son chevet (p. 5-6) ${ }^{6}$. C'est le même, pourtant, qui l'affuble plus tôt du surnom d' «abominable jésuite» (p. 3). Le passage est particulièrement intéressant parce qu'il place les enjeux de l'histoire précisément au cœur de la vie : au devoir de rupture d'avec le christianisme, qui, pour Joyce, paraît incompatible avec le réel, il oppose une éthique filiale renforcée par une mort qui s'avère éminente. L'auteur montre ainsi que le « cauchemar» de l'histoire ne peut se satisfaire d'une volonté

\footnotetext{
6 « Nom de Dieu, Kinch [surnom de Dedalus], vous auriez tout de même pu vous mettre à genoux quand votre mère mourante vous l'a demandé. Je suis un animal à sang froid comme vous. Mais penser que votre mère à son dernier soupir vous a supplié de vous agenouiller et de prier pour elle; et que vous avez refusé ! Il y a en vous quelque chose de démoniaque... ». Notons l'emploi du terme "démoniaque », qui fait basculer l'échec du devoir éthique dans une dénomination chrétienne dépréciative.
} 
d'oblitération dans la mesure où la condition dans laquelle il plonge le sujet est fort plus complexe que la dichotomie histoire/réel, devant notamment tenir compte des impératifs de l'intersubjectivité, et que, en outre, toute influence historique n'est pas forcément nuisible ou déréalisante.

Aussi cette expérience de l'histoire, telle que la conçoit Joyce, ne peut-elle se cantonner uniquement aux personnages. On peut citer à cet égard l'exemple de la devinette que pose Dedalus à ses élèves au chapitre II : «Le coq chantait, / Le ciel était bleu; / La cloche du bon Dieu / Sonnait onze heures. / Temps pour cette pauvre âme / De s'en aller aux cieux. ${ }^{7}$ » (p. 29) Tandis que les élèves marquent pour leur part leur incompréhension et ne proposent aucune solution en retour ${ }^{8}$, le lecteur est, indépendamment de leur réaction, sollicité lui aussi et appelé à trouver une réponse à l'énigme. Il appert que plusieurs signes tendent vers une lecture religieuse: «bon Dieu», " pauvre âme», "cieux», voire «ciel», "cloche» et «coq ». Le reniement de Pierre, tel que présenté dans l'Évangile de Luc, paraît dans cette optique comme une référence répondant bien dans l'ensemble aux signes proposés par Dedalus : «Jésus dit : "Je te le déclare, Pierre, le coq ne chantera pas aujourd'hui, que tu n'aies par trois fois nié de me connaître". » (p. 202) Sans entrer dans une exégèse excessive, car il y aurait beaucoup à dire ici, on note au moins deux éléments qui autorisent cette solution : le coq et la montée aux « cieux» du Christ. Certains l'invalident néanmoins : l'heure à

7 Les italiques sont de Joyce.

8 - Qu'est-ce? / - Quoi, monsieur? / - Encore, monsieur. On n'a pas entendu. / Leurs yeux s'ouvraient plus grands en écoutant une seconde fois les vers. Un silence et Cochrane dit: / - Qu'est-ce que c'est, monsieur? Nous donnons notre langue au chat. (p. 29) 
laquelle se déroule l'événement ( « ciel bleu » et « onze heures », qui s'opposent à la prophétie devant avoir lieu à l'aurore) et " [l]a cloche du bon Dieu » (qui pose certaines questions, ne pouvant évidemment renvoyer à une église chrétienne, mais pouvant toutefois être rattachée à une synagogue). En ce sens, la réponse que donne Dedalus, « [l] e renard enterrant sa grandmère sous un buisson de houx » (p. 29), plonge le lecteur, lui aussi, dans l'« espèce d'écho consterné » des « enfants » (p. 30). L'expérience de la devinette s'apparente, dans cette perspective, à celle qu'éprouvent les personnages envers l'histoire. En effet, l'histoire, en l'occurrence, permet d'expliquer; elle ne correspond, cela dit, qu'imparfaitement avec la devinette proposée. La réponse, elle, affirme sans ambages l'arbitraire d'une « historicisation » de l'énigme dans la mesure où elle met en avant la solution la plus imprévisible et contingente qu'il soit possible d'envisager. Ce faisant, personnage et lecteur ont des expériences qui se rejoignent.

\section{La réécriture comme expérience de l'histoire}

Si la devinette constitue un appel au lecteur, qui le plonge dans l'expérience trouble de l'histoire, force est de constater que la thématique de l'histoire se joue davantage, sinon quasi exclusivement, au sein du texte et, plus particulièrement, à travers la figure du personnage. Or, un aspect du roman semble, en l'occurrence, s'adresser plus particulièrement au lecteur. Il n'a en effet échappé à personne qu'Ulysse, par-delà l'intertextualité on ne peut plus foisonnante dont le roman témoigne, soit l'hypertexte de quelques livres, dont, au premier chef, 
l'Odyssée d'Homère. Le titre original de ce dernier livre, "Odysseus », signifie, soulignons-le, Ulysse, ce qui revient, pour Joyce, à donner pour titre à son roman celui-là même dont il découle. Pourtant, plusieurs exégètes, dont Gérard Genette dans Seuils, se questionnent sur la valeur réelle de cette réécriture : " [R]éduits à son seul texte et sans le secours d'aucun mode d'emploi, comment lirions-nous l'Ulysse de Joyce s'il ne s'intitulait pas Ulysse? » (p. 8) Ce que semble dire Genette est qu'il n'est pas sûr que, sous un autre titre, le roman ait jamais été mis en rapport avec le texte d'Homère. Pourtant, le titre ne fait à cet égard aucun doute : celui-ci agit à la manière d'un contrat de lecture avec le lecteur, qui, pour comprendre, est appelé à penser le roman à partir de l'hypotexte d'Homère. L'influence de l'Odyssée sur la civilisation occidentale (Fowler et al., 2004, p. 233-323) ne fait non plus aucun doute. S'il est un texte en effet qui est susceptible d'être connu du lecteur de Joyce, c'est bien l'Odyssée $e^{9}$. Lorsqu'on considère l'importance et la parenté des motivations des thématiques de l'histoire et de la réécriture, il est difficile de ne pas voir dans la dernière une extension de la première, destinée à faire de l'acte de lecture la manifestation des théories joyciennes de l'histoire. Dans cette hypothèse, l'hypotexte joue pour le sujet lisant le rôle de l'histoire pour le personnage. À la fois, l'hypotexte peut aider à la compréhension de l'hypertexte, il peut aussi projeter sur le roman de Joyce un sens qui ne s'y trouve pas : il convient moins en l'occurrence d'oblitérer l'hypotexte que de mettre en procès sa pertinence dans la préhension de l'hypertexte. Joyce force ainsi le lecteur à réfléchir aux présupposés de son expérience

\footnotetext{
9 Au reste, la critique a aussi relevé la présence d'Hamlet de Shakespeare comme autre hypotexte du roman, texte qui, on en conviendra, fait aussi partie des canons de la littérature universelle.
} 
de lecture. Tandis que la théorisation joycienne de l'histoire se présente grosso modo comme un refus de son influence sur le sujet, l'expérience de lecture anticipée par le roman viendrait à tout le moins mettre en cause le bien-fondé de cette influence et constituerait donc une amorce de réflexion à ce propos. La réécriture serait ainsi une possibilité romanesque instrumentalisée par les théories joyciennes sur l'histoire afin de faire de la relation du lecteur au texte un laboratoire expérimental du rejet de l'histoire. Dans Palimpsestes: la littérature au second degré, Gérard Genette définit l'hypertextualité comme «toute relation unissant un texte B ([...] hypertexte) à un texte antérieur A ([...] hypotexte) sur lequel il se greffe d'une manière qui n'est pas celle du commentaire » (p.11-12). Dans la définition extensive que donne Joyce de l'histoire, on pourrait, en reprenant la terminologie de Genette, associer cette dernière à une instance $\mathrm{B}$, ayant une influence sur une instance A, qui serait le réel. Forcément, il faudrait, dans cette comparaison, rajouter une instance $\mathrm{C}$, dans le cas de la réécriture, le lecteur, dans celui de l'histoire, le sujet ou, plus précisément, le personnage : cette instance $\mathrm{C}$ serait la médiatrice des relations $\mathrm{AB}$.

Hormis la popularité du texte, qui facilite la réflexion sur la relation $A B$ chez le lecteur empirique $C$, la question du pourquoi de l'Odyssée comme principal hypertexte du roman se pose toujours. Plusieurs hypothèses ont été proposées, ce qui a brièvement été rappelé en introduction. La mise en relation des thématiques de l'histoire et de la réécriture permet cependant, il nous semble, de nouvelles perspectives sur cette question. Dans De l'utilité et de l'inconvénient des études historiques pour la vie, dont Robert Spoo a montré que le texte était dans une certaine mesure le point de départ de la réflexion de Joyce sur 
l'histoire, Nietzsche rappelle que la civilisation grecque a "longtemps été un chaos d'influences étrangères, sémitiques, babyloniennes, lydiennes [et] égyptiennes, tant sur le plan de la forme que des idées, et que leur religion [était] véritablement une bataille entre dieux», mais que cette civilisation a "progressivement appris à organiser [ce] $\operatorname{chaos}^{10}$ ». Si une exégèse exhaustive des implications de cette perspective sur la civilisation hellène ne peut avoir lieu ici, notons tout de même qu'en tant que cette civilisation serait celle qui serait passée du «chaos» à une «organis[ation]» pacifique des diverses influences entre elles, elle pourrait apparaître à Joyce comme un modèle en la matière. Ce serait notamment en ce sens que pourrait être interprétée la pensée de Buck Mulligan au tout début du roman: «[S]i seulement nous pouvions travailler ensemble, nous ferions quelque chose pour notre île. L'helléniser. " (p. 7) Certes, il y aurait beaucoup à dire du pourquoi de la présence de l'hypotexte d'Homère. Or, en l'état, sa présence appert comme paradoxale. En effet, l'hypotexte (A) lui-même semble thématiquement se présenter comme un modèle d'inclusion des diverses influences historiques, qui permettrait d'endiguer les effets délétères de l'histoire. Cela dit, la relation $\mathrm{AB}$, puisqu'imparfaite, viendrait questionner la pertinence de l'instance $A$ dans la compréhension de l'instance B pour le lecteur (C). L'hypotexte (A) serait ainsi le

\footnotetext{
${ }^{10}$ Je traduis : « [T] he Greek culture of antiquity was "for a long time a chaos of foreign, Semitic, Babylonian, Lydian, [and] Egyptian forms and ideas, and their religion [was] truly a battle of all the gods of the East." The Greeks, however, "gradually learned to organize the chaos" by distinguishing their true needs from the false ones [...] ». Nacona Nix, 2008, p. 47, se référant à Nietzsche, On the Uses and Disadvantages of History for Life, 1997, p. 122. Les passages cités et traduis sont exclusivement tirés de ce dernier livre de Nietzsche.
} 
double signe des influences inclusives et tyranniques de l'histoire.

\section{La nostalgie du corps comme symbole}

Bien qu'elle se soit arrêtée sur quelques passages ponctuels, l'analyse a jusqu'ici abordé les thématiques de la réécriture et de l'histoire "de haut ». Notre but est de terminer cette étude de manière plus ciblée par l'analyse de la réécriture de la thématique de la nostalgie et, plus particulièrement, de l'épisode que le schéma Linati intitule "Ithaque», au cours duquel se clôt la quête "des Ulysses », en tentant de montrer que la parodie de la notion de nostalgie vient, en explicitant une critique de la notion d'histoire, mettre en avant un nouveau rapport du sujet au monde. L'équivalent hypotextuel de cet épisode se trouve à la fin de l'Odyssée, lors du retour à Ithaque qu'Ulysse, malgré la nostalgie diffuse qu'il éprouve tout au long de ses pérégrinations, ne reconnaît pas :

[...] Ulysse s'éveillait de son premier sommeil sur la terre natale, mais sans la reconnaître après sa longue absence; car Pallas Athéna, cette fille de Zeus, avait autour de lui versé une nuée, afin que, de ces lieux, il ne reconnût rien et qu'il apprît tout d'elle [...]. Aussi, devant les yeux du maître, tout n'était que des sites étrangers, les mouillages des ports, les rocs inaccessibles, les sentes en lacet et les arbres touffus. (1999, p. 247)

Pénélope, souvenons-nous, ne reconnaît pas non plus d'emblée l'amant qu'elle a attendu pendant vingt ans. L'objet de la nostalgie d'Ulysse est double : Ithaque et Pénélope. Pourtant, il ne reconnaît pas Ithaque et Pénélope ne reconnaît pas Ulysse. 
Homère introduit ainsi une donnée importante dans la réflexion

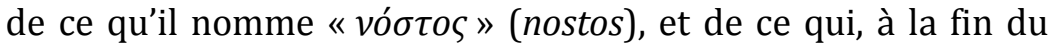
XVIIe siècle, sera par la suite appelé " nostalgie " ${ }^{11}$, à savoir le Temps. Tandis que les voyages d'Ulysse mettent en avant la spatialité de la nostalgie, le retour ou nostos montre que la nostalgie se décline au premier chef dans le cadre d'une temporalité qui détermine ses différents aspects. Ulysse a en effet vieilli et Ithaque, changé, sous l'impulsion du temps, qui place les rencontres du retour sous le signe de l'inconnaissance.

A contrario, les composantes tant spatiale que temporelle sont déniées à la nostalgie dans Ulysse de Joyce : le roman se décline dans les cadres étriqués d'une ville - Dublin - et d'à peine une journée - 18 heures. À la Méditerranée, qui est la limite spatiale des voyages d'Ulysse, Joyce substitue une ville; il remplace les vingt années par dix-huit heures. De plus, tandis que le lit, chez Homère, est le signe ultime de la réunion des amants, lorsque le héros de Joyce, Léopold Bloom, se glisse sous les couvertures de son lit, dans l'épisode intitulé «Ithaque», il ne trouve pas son amante Molly Bloom, mais plus précisément, comme l'écrit Joyce, son «corps» endormi : «Que rencontrèrent ses membres en s'étendant graduellement? / De nouveaux draps, propres; des odeurs supplémentaires; la présence d'un corps humain, féminin, le sien à elle [...]. ${ }^{12}$ » (1922, p. 794) Notons d'abord l'emploi des pronoms possessif et personnel "sien » et "elle », qui vise à établir une distance entre le «corps» endormi et Molly Bloom. Le monologue intérieur de Molly Bloom, qui clôt le roman, se focalise d'autre

\footnotetext{
11 « [...] 1688, the year of the nostalgia's linguistic birth. A compound made up of nostos, meaning "journey" or "journey home," and algia, "pain," nostalgia is not and simply could not be a Greek word. » (Illbruck, 2012, p. 5) 12 Je souligne.
} 
part presque exclusivement sur le corps féminin comme objet de désir. C'est donc dire que, là où Homère faisait de la réunion des êtres la téléologie de la nostalgie d'Ulysse, la finalité de son équivalent joycien, Léopold Bloom, dont les dix-huit heures de pérégrinations annihilent la pertinence de toute reconnaissance, serait celle du corps, dont la «présence» seule permettrait l'identification de Molly Bloom.

Par-delà la parodie évidente d'Homère en l'occurrence, l'identification du noème de la nostalgie au corps pose la question de la fonction possible de ce dernier dans le roman. Une historicisation du corps paraît, pour ce faire, nécessaire. Il faut, dans cette optique, vraisemblablement remonter aux libertins du XVIII siècle, qui font du corps un instrument de contestation du dogme chrétien (Trousson, 1993). S'attaquant lui aussi au premier chef à la « morale » chrétienne (Nietzsche, 1887), Nietzsche fait du corps un instrument de réhabilitation du réel (Valadier, 1968; Blondel, 1986, p. 87-88; Delfour, 2014). Le corps, ayant en soi été largement nié dans l'histoire sous le joug de l'Église catholique (Corbin, Courtine, Vigarello et al., 2005, p. 50-83; Andlauer, 2008; Arnold, $1994^{13}$ ), devient, sous l'impulsion de Nietzsche, le signe privilégié d'un ancrage au réel. Étant donné la prégnance de la réflexion sur l'histoire et sur la possibilité des personnages d'accéder au réel, l'identification de la quête de Bloom au corps, paraît, à travers l'historicisation que nous en avons fournie, ne pas être anodine. L'hypothèse que fait Maja Herman-Sekulid, qui se propose de penser la réécriture d'Ulysse à partir de la notion d'

13 Relevons en outre cette citation d'Alphonse de Liguori, docteur de l'Église du XVIII e siècle, intercalée dans Le Corps et l'âme d'Odile Arnold : " Le plus grand de nos ennemis, c'est notre corps. » (1994, p. 136) 
« hyperbate» $\left(1986\right.$, p. $\left.4^{14}\right)$, semble ici pouvoir fournir un cadre théorique idoine aux motivations de la réécriture ponctuelle de la nostalgie. Herman-Sekulid conceptualise la notion d'«hyperbate », d'ordinaire définie comme une figure de style consistant à séparer deux mots que l'usage regroupe, comme «l'interversion de la dissolution [du texte] par [s]a reconstruction » $\left(1986\right.$, p. $\left.87^{15}\right)$. La nostalgie joycienne du corps paraît en effet relever du double impératif de dissolution et de reconstruction du sens. D'abord, la nostalgie, chez Homère, renvoie à des temporalité et spatialité longues que la ville unique et les dix-huit heures dans lesquelles Joyce situe Ulysse viennent en quelque sorte "dissoudre» dans une ironie évidente; l'identification de son noème aux êtres et à la terre natale chez Homère, au corps chez Joyce, finit cette « dissolution ». L'analyse comparée de la thématique de la nostalgie semble donc se solder par une incompatibilité aporétique tant Joyce paraît avoir dégagé la thématique originelle de son contenu profond. Or, l'identification de la nostalgie de Bloom au corps présente, semble-t-il, une « reconstruction », précisément, du sens. Il a déjà été montré, tout au long de cette étude, la manière dont la conception joycienne de l'histoire s'inspire de celle de Nietzsche. Au demeurant, l'identification de la nostalgie au corps peut, en ce sens, être pensée, dans l'idée dont Nietzsche s'en fait, comme une quête de ce qui permet un rapport authentique à la vie. L'ancrage au corps serait ce qui permettrait de médiatiser l'histoire, et autoriserait ce qu'oblitère cette dernière, le lien au réel. L'épisode d'«Ithaque » serait donc la figure, la métaphore d'une téléologie de la vie, d'un rapport de préhension authentique à la vie, tandis que cette quête elle-même, bien que dérisoire au vu de

\footnotetext{
14 Je traduis : " hyperbaton».

15 Je traduis : «[...] the inversion of dissolution into reconstruction ».
} 
la parodie à laquelle elle est sujette, serait néanmoins et précisément l'explicitation de cet impératif de l'ancrage au corps, et à travers lui, au réel. Tout écart entre hypotexte (A) et hypertexte $(\mathrm{B})$ ne serait donc pas destiné uniquement à mettre en procès la relation $A B$ et, par extension, pour le lecteur, de mettre en cause toute influence «historique» dans sa préhension du réel, mais pourrait aussi, comme l'ont proposé certains critiques, et comme nous le proposons à notre tour, expliciter un sens neuf qui s'érigerait sur les décombres du sens « dissolu ». Toute la complexité de la thématique de la réécriture chez Joyce, il faut en convenir, se joue là : dans la pluralité de ses emplois et possibles, constitutive du classique constitué qu'est Ulysse.

\section{Bibliographie}

ANDlAUER, Jeanne. (2008), Modeler des corps. Reliquaires, canivets et figures de cire des religieuses chrétiennes, Lille, Atelier national de reproduction des thèses.

ARnold, Odile. (1984), Le Corps et l'âme, Paris, Seuil.

BLONDEL, Éric. (1986), Nietzsche, le corps et la culture: la philosophie comme généalogie philosophique, Paris, Presses universitaires de France.

Corbin, Alain, Jean-Jacques Courtine, Georges Vigarello et al. (2005), Histoire du corps, t. II, Paris, Seuil.

DELFour, Jean-Jacques. (2006), "Continuité et discontinuité dans la critique nietzschéenne de la métaphysique. Réflexions sur les "contempteurs du corps" dans Ainsi parlait Zarathoustra », Philopsis, <http://www.philopsis.fr/spip.php?article26>. 
EllmanN, Richard. (1962), "Ulysses and the Odyssey », English Studies, vol. 43, p. 423-426.

FAIRHALL, James. (1993), James Joyce and the Question of History, Cambridge et New York, Cambridge University Press.

Fowler, Robert et al. (2004), The Cambridge Companion to Homer, Cambridge, Cambridge University Press.

STANFORD FRIEDMAN, Susan (dir.), (1993), Joyce: The Return of the Repressed, Londres, Cornell University Press.

FroulA, Christine. (1991), "History's Nightmare, Fiction's Dream: Joyce and the Psychohistory of Ulysses », James Joyce Quarterly, vol. 28, no 4, p. 857-872.

GenetTe, Gérard. (1982), Palimpsestes : la littérature au second degré, Paris, Seuil.

-. Seuils. (1987), Paris, Seuil.

Herman-SeKulid, Maja. (1986), The Fall of Hyperbaton: Parodic and Revisionary Strategies in Bely, Joyce and Mann, Princeton, Princeton University.

Homère. (1999), Odyssée, Paris, Gallimard, coll. «Folio classique ».

ILLBRUCK, Helmut. (2012), Nostalgia: Origins and Ends of an Unenlightened Disease, Evanston, Northwestern University Press.

JoyCE, James. (1995 [1922]), «Ulysse », dans OEuvres, t. II, Paris, Gallimard, coll. « Bibliothèque de la Pléiade ».

LEVIN, Harry. (1960), James Joyce, New York, New Directions.

LEVITT, Morton. (1999), James Joyce and Modernism: Beyond Dublin, Lewiston et New York, Edwin Mellen Press. 
MADTES, Richard. (1983), The 'Ithaca' Chapter of Joyce's "Ulysses", Ann Harbour, UMI Research Press.

NADEL, Ira. (1989), Joyce and the Jews, Iowa City, University of Iowa Press.

NiETzSCHE, Friedrich. (1964), Considérations inactuelles, Paris, Aubier-Montaigne.

—. (1900 [1887]), Généalogie de la morale, Paris, Mercure de France.

-. (1997), On the Uses and Disadvantages of History for Life, dans Untimely Meditations, Cambridge, Cambridge University Press.

NIX, Nacona. (2008), Nietzsche's Historiography: History and Culture in the Second Untimely Meditation, thèse de doctorat, Université de Caroline du Nord.

NoRRIS, Margot. (1998), A Companion to Joyce's Ulysses, Boston, Bedford / St. Martin.

Pound, Ezra. (1968), Literary Essays of Ezra Pound, New York, New Directions.

SP00, Robert. (1994), James Joyce and the Language of History, New York et Oxford, Oxford University Press.

STANFoRD, W. B. (1953), «Ulyssean Qualities in Leopold Bloom », Comparative Literature, vol. 5, no 2, p. 125-136.

TAGoloupos, Constance. (1992), "Joyce and Homer: Return, Disguise, and Recognition in 'Ithaca' », dans John McCourt (dir.), Joyce in Context, Cambridge, Cambridge University Press, p. 184-200.

Trousson, Raymond et al. (1993), Romans libertins du XVIIIe siècle, Paris, Robert Laffont.

VALADIER, Paul. (1974), Nietzsche et la critique du christianisme, Paris, Éditions du Cerf. 


\title{
Résumé
}

Cet article a pour but de montrer la manière dont la thématique de l'histoire permet de réfléchir à celle de la réécriture dans Ulysse de James Joyce. En effet, si le roman présente l'histoire comme une influence délétère pour le sujet, lui interdisant un rapport de préhension authentique au réel, il appert que l'Odyssée d'Homère joue, dans l'optique de la compréhension du roman, un rôle similaire. Il s'agira donc de conceptualiser à grands traits la transposition de la thématique de l'histoire au sein de l'expérience de lecture.

\begin{abstract}
This article attempts to show how the theme of history allows to reflect on the rewriting in Ulysses of James Joyce. In fact, if the novel presents history as a deleterious influence for the subject, prohibiting it an authentic prehension link to reality, it appears that Odyssey of Homer plays a similar role in the understanding of the novel. This article will therefore try to conceptualize briefly the transposition of the thematic of history into the reading experience.
\end{abstract}

